\title{
Clinical Progress Notes: Updates from the 4th Universal Definition of Myocardial Infarction
}

\author{
Christopher J King, MD*; Andrew E Levy, MD²; Jeffrey C Trost, MD³
}

'Department of Medicine, Division of Hospital Medicine, University of Colorado, Aurora, Colorado; ${ }^{2}$ Department of Medicine, Division of Cardiology, University of Colorado, Aurora, Colorado; ${ }^{3}$ Department of Medicine, Division of Cardiology, Johns Hopkins University, Baltimore, Maryland.

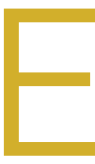

levated serum troponin clearly does not equal myocardial infarction (MI). This was the strong message in the 2018 publication of the Fourth Universal Definition of Myocardial Infarction ${ }^{1}$ (4UDMI), the first update to the international consensus document since 2012.

Most clinicians have learned how to accurately diagnose the classic Type $1 \mathrm{MI}$ (T1MI) due to atherosclerotic plaque rupture; however, elevated troponin in the absence of T1Ml is increasingly common due to more frequent and less discriminate troponin testing. ${ }^{2}$ Patients with elevated troponin in the absence of T1MI have traditionally created confusion and variability in diagnosis, management, and documentation. Interpretation and management of elevated troponin in the absence of T1Ml has become difficult.

In this clinical practice update, we aim to review the updated definition of Type $2 \mathrm{MI}$ (T2MI) and nonischemic myocardial injury (NIMI), since these are the two predominant diagnoses among patients with elevated troponin in the absence of T1MI. We also provide a clinical framework for clinicians to think through elevated serum cardiac troponin levels and identify opportunities for quality improvement around this critical issue.

\section{DEFINITIONS OF MYOCARDIAL INJURY}

The presence of an elevated serum troponin level is a critical component in determining the presence of cardiac myocyte injury and possible infarction. Myocardial injury is defined as the presence of serum troponin above the 99th percentile of the upper reference limit (URL), the absolute value of which varies by assay and which applies to traditional and highly sensitive subtypes. Myocardial injury can be confusing to assess, as it can be acute or chronic.

When troponin levels are elevated but stable, this is in dicative of chronic (usually nonischemic) myocardial injury, as seen, for example, in patients who have end-stage renal disease. The presence of acute injury requires a change in the troponin value-specifically a rise and/or fall in troponin levels with serial measurements. What constitutes a significant "rise and/or fall" is a matter of some debate and is not precisely defined in the $4 U D M I$. The percent change in the troponin value over time (relative delta) is listed as part of

*Corresponding Author: Christopher J. King, MD; E-mail: christopher.king@ ucdenver.edu; Telephone: 720-848-4289; Twitter: @cjking38.

Published online first August 21, 2019

Received: May 3, 2019; Revised: July 1, 2019; Accepted: July 7, 2019

(c) 2019 Society of Hospital Medicine DOI 10.12788/jhm.3283 the criteria for acute injury when the change is greater than or equal to $20 \%{ }^{1}$ however, clinicians should be aware that absolute delta in troponin (the change in $\mathrm{ng} / \mathrm{dL}$ ) has better performance characteristics ${ }^{3}$ in diagnosing acute myocardial injury. Regardless of whether clinicians use relative or absolute changes in the serum troponin level, clinical evaluation of patients with acute injury is critical to establishing whether the injury is ischemic (MI) or nonischemic (NIMI). The presence of at least one of the following is necessary to meet the current criteria for myocardial ischemia according to the fourth universal definition: new ischemic symptoms (eg, chest pain, dyspnea, etc.), new ischemic changes in the patient's electrocardiogram (eg, new ST segment depression in leads $\mathrm{II}, \mathrm{II}$, and aVF), or cardiac imaging changes consistent with ischemic injury (eg, new wall motion abnormality in the inferior wall on echocardiography).

Following diagnosis of Ml based on elevated troponin and new symptoms or signs, the cause of $\mathrm{Ml}$ should then be determined. Type $1 \mathrm{Ml}$ remains defined as $\mathrm{Ml}$ caused by atherosclerotic plaque disruption in a patient with coronary artery disease (CAD). Type $2 \mathrm{Ml}$ is not caused by plaque disruption but is due to a mismatch between oxygen supply and demand unrelated to acute atherothrombosis. T2Ml is an ischemic myocardial injury traceable to some other illness that leads to inadequate myocyte oxygenation. Causes of T2Ml are numerous, can overlap with nonischemic injury, and can include severe anemia, septic shock, rapid atrial fibrillation, and coronary dissection. While CAD may be present in patients with T2MI, it is not a requirement, and an increased demand for, or reduced supply of, myocyte oxygen alone can be sufficient to cause MI.

In the absence of clinical signs or symptoms of cardiac ischemia, clinicians should categorize patients as having a nonischemic myocardial injury. There is significant overlap between causes of T2MI and NIMI, for example, sepsis could cause either T2MI or NIMI. What distinguishes these two entities is whether the signs and symptoms for myocardial ischemia as outlined above are present. If these signs or symptoms are present, the diagnosis is T2MI. If no clinical signs or symptoms of ischemia are present, the diagnosis is NIMI. The assessment of the clinician, using all available clinical information, is pivotal. The characteristics of the three major types of myocardial injury are depicted in the Figure.

\section{CLINICAL PRACTICE UPDATE}

Proper distinction between infarction or injury without infarction is central to proper evaluation, treatment, and eventual docu- 


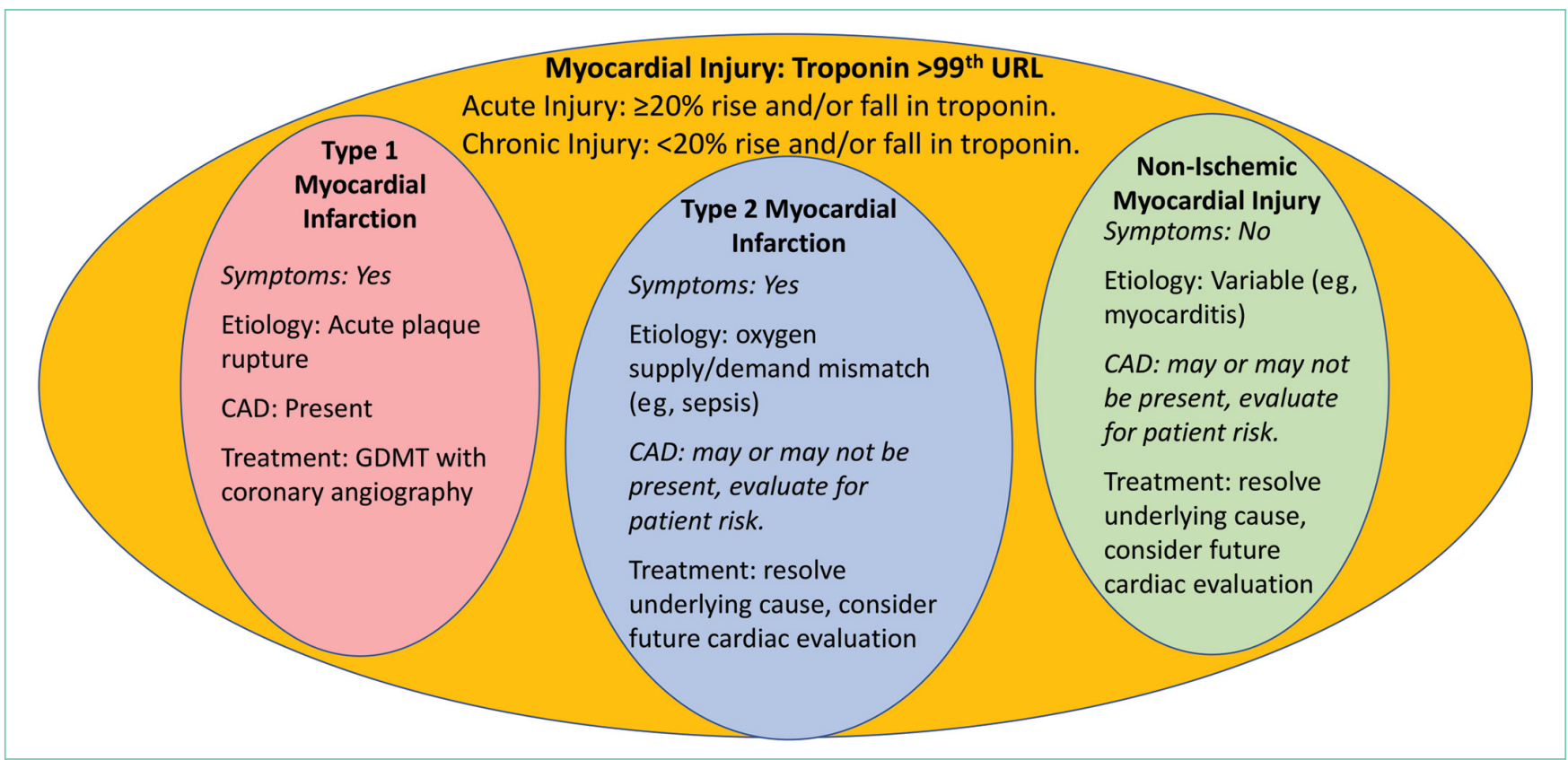

FIG. Differing Characteristics of Common Causes of Myocardial Injury. Components Directly Requiring the Provider's Clinical Judgment are in Italics.

Abbreviations: CAD, coronary artery disease; GDMT, guideline directed medical therapy; URL, upper reference limit.

mentation in patients with elevated troponin levels. In the case of T2MI and NIMI, identifying what underlying illness is causing the troponin elevation is essential for acute management.

\section{Evaluation}

Troponin elevation is associated with an elevated risk for major adverse cardiovascular events, regardless of etiology. ${ }^{4}$ While patients with suspected T1MI are most often evaluated by coronary angiography, this may not be necessary for patients with T2MI or NIMI. Developing an evaluation strategy for patients with $\mathrm{T} 2 \mathrm{MI}$ or NIMI requires understanding the underlying etiology of myocardial injury. In patients with septic shock for example, there are many potential mechanisms for cardiac myocyte injury, many of which are nonischemic (eg, cytokine-mediated). ${ }^{5}$ Prompt evaluation and treatment of septic shock, therefore, often leads to resolution of cardiac dysfunction, and ischemic evaluation may not be necessary. ${ }^{6}$ In many cases of $\mathrm{T} 2 \mathrm{Ml}$ or NIMI, waiting for an acute underlying illness to resolve is necessary before deciding whether ischemic evaluation is appropriate. It is important that this decision is deferred but not forgotten though as patients with $\mathrm{T} 2 \mathrm{MI}$ or NIMI may benefit from further cardiac evaluation. There are no society recommendations and minimal evidence to guide this evaluation, but clinical trials testing different evaluation strategies are underway. ${ }^{7}$ Until an optimal evidence-based evaluation strategy becomes clear, clinicians should focus on two key principles: first, determine and treat the underlying etiology; second, identify patients with traditional risk factors for CAD and consider further evaluation with either coronary angiography or cardiac imaging. Referral to a cardiologist for assistance with the latter issue, especially for challenging or equivocal cases, is encouraged.

\section{Treatment}

While T1MI therapies have a strong evidence base with high rates of appropriate treatment, there are relatively few evidence-based therapies for T2MI and NIMI. The benefits of traditional T1Ml therapies should be considered in terms of each therapy's risk-benefit profile. Among patients with T2MI or NIMI in whom atherosclerotic plaque rupture is unlikely, or in whom bleeding risk is high, antithrombotic agents such as unfractionated heparin and dual antiplatelet therapy represent low value and potentially harmful therapies. ${ }^{8}$ Conversely, patients with multiple risk factors for CAD may benefit from low-risk guideline directed medical therapies such as HMGCoA reductase inhibitors (ie, "statins"). Recent data suggest that lipid-lowering therapies may even be beneficial for preventing T2MI. ${ }^{9}$

Given the lack of evidence for therapies to treat patients with $\mathrm{T} 2 \mathrm{MI}$ or $\mathrm{NIMI}$, clinical judgment remains central to creating an optimal management plan. Clinicians should consider consultation with a cardiologist any time there is ambiguity in whether the diagnosis is T1MI or T2MI. For example, postoperative patients represent a particularly challenging clinical scenario due to the difficulty of assessing ischemic signs and symptoms in the operating room. In this setting, early evaluation by a cardiologist has been shown to improve outcomes. ${ }^{10}$

\section{Documentation}

Documentation of non-ST elevation MI (NSTEMI) for every case of elevated troponin, rather than using the more specific T1MI, T2MI, or NIMI terminology, can have adverse consequences for health systems. From a coding perspective, the terms STEMI and NSTEMI mean T1MI, and the ICD-10 codes used to identify $\mathrm{T} 1 \mathrm{Ml}$ patients for value-focused programs frequently include patients with T2MI and NIMI due to impre- 
cise documentation. ${ }^{11}$ When T2MI and NIMI are imprecisely documented as NSTEMI, health systems and clinicians are held to the T1Ml care standards. This can negatively skew the performance of a health system or individual clinician because T2MI and NIMI patients have worse outcomes than T1MI patients. ${ }^{4}$ Inaccurate categorization of patients can lead to inaccurate quality and registry reporting, which may hinder the ability of health systems to monitor and implement quality improvement programs for $\mathrm{Ml}$ patients. The distinction between $\mathrm{T} 1 \mathrm{Ml}$ and $\mathrm{T} 2 \mathrm{Ml}$ in documentation is all the more important now that a new ICD-10 code exists for T2MI (I21. A1), which allows clinicians to more precisely identify these patients, both clinically and administratively, as distinct from T1MI patients. ${ }^{12}$ While there is no similarly specific ICD-10 code for $\mathrm{NIMI}$, using the appropriate terminology in documentation should prompt coding personnel to use a code for "other abnormal findings of blood chemistry," reflecting cardiac biomarker elevation (R79.89), rather than using one of the T1Ml codes. Clinicians may not be able to determine the etiology of troponin elevation in the initial phase of a hospitalization, but a definitive diagnosis should be documented in the discharge summary.

From the patient perspective, documentation using STEMI and NSTEMI can mislead clinicians, given that this terminology does not specify the underlying cause (ie, plaque rupture or oxygen supply-demand mismatch), potentially leading to delayed initiation of appropriate therapy. Incorrect documentation, using STEMI/NSTEMI language or incorrectly labeling T2MI and NIMI, may lead patients to believe they have had a heart attack when they had myocardial injury instead. This may lead to unnecessary anxiety and change their interactions with the health system. These patients may be started on unnecessary therapies, have inaccurate preoperative evaluations, and be labeled with a preexisting condition for the rest of their lives.

\section{Opportunities for Quality Improvement}

Systems-based quality improvement can help to ensure that patients with NIMI and $\mathrm{T} 2 \mathrm{MI}$ are labeled appropriately and receive the proper treatment. Clinical education is the cornerstone of such efforts. Hospital medicine practitioners looking for support in understanding this issue might first partner with cardiology colleagues who may already have training materials available. Billing and coding personnel stand to benefit from greater orientation to this topic considering the complexities and errors in the coding of MI. In an attempt to help nonclinical coding personnel, leaders in the American College of Cardiology have advocated for use of the term "non-Ml troponin elevation" (nMITE) ${ }^{13}$ to describe NIMI patients. Finally, we see an important role for EHR-embedded decision support tools to facilitate appropriate documentation and management of patients with elevated troponin. A potential care pathway, for example, could be created in partnership between hospital medicine and cardiology providers to identify which patients would benefit from cardiologist consultation.

\section{CONCLUSIONS}

Understanding the definitions of $\mathrm{T} 1 \mathrm{MI}, \mathrm{T} 2 \mathrm{MI}$, and $\mathrm{NIMI}$ will help clinicians to better identify the appropriate clinical care and consultation strategy for patients with elevated cardiac troponin. There are relatively few published quality improvement initiatives to help guide clinicians through these nuanced distinctions, but there is great potential in such approaches to help clinicians provide the highest value care possible.

Disclosures: No authors have any conflict of interest, financial or otherwise, to declare regarding this study.

Funding: Dr. Levy receives funding from National Institutes of Health (NIH) T32 Training Grant 5T32-HL007822.

\section{References}

1. Thygesen K, Alpert JS, Jaffe AS, et al. Fourth universal definition of myocardial infarction (2018). J Am Coll Cardiol. 2018;72(18):2231-2264. https://doi. org/10.1016/j.jacc.2018.08.1038.

2. Shah ASV, Sandoval Y, Noaman A, et al. Patient selection for high sensitivity cardiac troponin testing and diagnosis of myocardial infarction: prospective cohort study. BMJ. 2017;359:j4788. https://doi.org/10.1136/bmj.j4788.

3. Storrow AB, Nowak RM, Diercks DB, et al. Absolute and relative changes (delta) in troponin I for early diagnosis of myocardial infarction: results of a prospective multicenter trial. Clin Biochem. 2015;48(4-5):260-267. https://doi. org/10.1016/j.clinbiochem.2014.09.012.

4. Sandoval $Y$, Jaffe AS. Type 2 myocardial infarction. J Am Coll Cardiol. 2019;73(14):1846-1860. https://doi.org/10.1016/j.jacc.2019.02.018.

5. Martin L, Derwall M, Al Zoubi S, et al. The septic heart: current understanding of molecular mechanisms and clinical implications. Chest. 2019;155(2):427437. https://doi.org/10.1016/j.chest.2018.08.1037.

6. Vallabhajosyula S, Jentzer JC, Geske JB, et al. New-onset heart failure and mortality in hospital survivors of sepsis-related left ventricular dysfunction. Shock. 2018:49(2):144-149. https://doi.org/10.1097/SHK.0000000000000952.

7. Lambrakis K, French JK, Scott IA, et al. The appropriateness of coronary investigation in myocardial injury and type 2 myocardial infarction (ACT2): a randomized trial design. Am Heart J. 2019;208:11-20. https://doi. org/10.1016/j.ahj.2018.09.016.

8. Morrow A, Ahmad F, Steele C, McEntegart M, Murdoch D. Treating the troponin: adverse consequences of over-treatment of elevated troponin in non-coronary presentations. Scot Med J. 2019;64(1):10-15. https://doi. org/10.1177/0036933018809754.

9. White HD, Steg P, Szarek M, et al. Reduction of type 1 and type 2 myocardial infarctions in patients treated with alirocumab: insights from the ODYSSEY Trial. J Am Coll Cardiol. 2019;73(9):4. https://doi.org/10.1016/S07351097(19)30613-8.

10. Hua $A$, Pattenden $H$, Leung $M$, et al. Early cardiology assessment and intervention reduces mortality following myocardial injury after non-cardiac surgery (MINS). J Thorac Dis. 2016;8(5):920-924. https://doi.org/10.21037/ jtd.2016.03.55

11. Díaz-Garzón J, Sandoval Y, Smith S, et al. Discordance between ICD-coded myocardial infarction and diagnosis according to the universal definition of myocardial infarction. Clin Chem. 2017;63(1):415-419. https://doi. org/10.1373/clinchem.2016.263764.

12. Goyal A, Gluckman TJ, Tcheng JE. What's in a name? The new ICD-10 (10th revision of the International Statistical Classification of Diseases and Related Health Problems) codes and type 2 myocardial infarction. Circulation. 2017;136(13):1180-1182. https://doi.org/10.1161/CIRCULATIONAHA.117.030347.

13. Goyal A GT, Levy AE, Mariani D, et al. Translating the fourth universal definition of myocardial infarction into clinical documentation. Cardiology. 2018:34-36. 